\title{
THE WORK BEHIND THE SCENES
}

Viebig, RG. The work behind the scenes. Arq Gastroenterol. 2014;51(1):2-3.

HEADINGS - Peer review.

A well-produced scientific journal in Brazil is an insane act of science and teaching love. Only the passion can explain the existence of a periodical, in this case the Archives of Gastroenterology, for more than 50 years. Working in a small room, previously among piles of papers and typewriter machines now, in front of electronic paraphernalia, nothing has changed the attitude of the people involved in this process. All individuals involved in editorial work, making it for some reason, but never for notoriety, financial reward or for other reasons of a self-centered, once operate behind the scenes. On editor role, I feel just as an interlocutor among the authors and the most important figure for the production of our journal: the Reviewer.

From the beginning, their work occurs in the anonymity and willingness. Nobody influences in your judgment or opinion, since this opinion is based on their degree of knowledge and the will to help the author(s) to produce a quality text or a more refined search. Without the person of the anonymous reviewer a scientific journal is a publication without credibility, without criteria, without soul. The reviewers are responsible for the scientific journal profile, doing it deeper, more critical and more current, especially in Archives, which aim to publish topics regarding the different areas of the largest specialty within Medicine. Unquestionably, the range of topics and variables within the areas of specialization of Gastroenterology, make the Archives rich in sources of research, which implies the increase of their responsibility in the contents. Our immediate rejection index gets close to $50 \%(2011,2012$ and 2013), since it leads us to two interpretations: either our criteria for publishing a work are rigid, or serves as an inhibitor factor for sending jobs with poor potential for publication, in the recognition of the journal seriousness.

We have the point of view that active reviewers of a periodical, admittedly important should be graced somehow by the academic and scientific community. We are someway trying to change this situation but it demands a lot of time and patience. We are grateful for his work and goodwill, and recognize, once again, its importance to the existence of the Archives of Gastroenterology. Each year we publish a list of acknowledgments at the end of the journal, and now, will anticipate demonstrating our gratitude.
Many thanks to all contributors of the journal and if someone has been omitted, we apologize in advance and please let us know.

Ricardo Guilherme VIEBIG*

Almeida, José Roberto de

Alves, Paulo Roberto Arruda

Alves, Venancio Avancini Ferreira

Amaral, Sanzio Santos

Andreoli, João Carlos

Andreollo, Nelson Adami

Araujo, Sérgio Eduardo Alonso

Ardengh, José Celso

Averbach, Marcelo

Azzam, Rimon Sobhi

Bahia, Magda

Barbosa, Alfredo J. Afonso

Begnami, Maria Dirlei Ferreira de Souza

Bittencourt, Paulo Lisboa

Borini, Paulo

Bottoni, Andrea

Brandão, Ajácio Bandeira de Mello

Brandão, Maria Angela Bellomo

Brasil, Horus Antony

Burini, Roberto Carlos

Campos, Fabio Guilherme

Caporossi, Cervantes

Cappellanes, Carlos Alberto

Carrilho, Flair José

Catapani, Wilson Roberto

Cecconello, Ivan

Chaves, Dalton Marques

Chinzon, Decio

Cleva, Roberto

Cliquet, Marcelo Gil

Coelho, Henrique Sérgio Moraes

Coelho, Júlio Cezar Uili

Coelho, Luis Gonzaga Vaz

Collares, Edgard

Costa, Milton M. Barbosa

Cotrim, Helma Pinchemel

Coy, Claudio Saddy Rodrigues

Cuenca, Ronaldo Mafia

Cunha, José Eduardo Monteiro da

Cyrillo, Marcos Antonio

*Executive Editor 
D’Albuquerque, Luiz Augusto Carneiro

Damião, Aderson Omar

Dantas, Roberto Oliveira

Domingues, Gerson Ricardo de Souza

Eisig, Jaime Natan

Fagundes Neto, Ulysses

Faintuch, Joel

Farias, Alberto Queiroz

Ferrari Jr, Angelo Paulo

Ferraz Neto, Ben Hur

Ferraz, Alvaro Antonio Bandeira

Ferraz, Edmundo Machado

Forones, Nora Manoukian

Furkim, Ana Maria

Garrido Jr, Arthur Belarmino

Guarita, Dulce Reis

Haddad, Luciana

Herbella, Fernando A. M.

Herman, Paulo

Jacob, Carlos Eduardo

Jorge, José Márcio Neves

Kadowaki, Norma Terumi

Kawakami, Elisabete

Kemp, Rafael

Koda, Yu Kar Ling

Kotze, Lorete Maria da Silva

Kotze, Paulo Gustavo

Lemme, Eponina Maria Oliveira

Lopasso, Fabio Pinatel

Lopes Filho, Gaspar de Jesus

Lopes, César Vivian

Lopes, Edmundo Pessoa

Machado, Marcel Autran Cesar

Machado, Rodrigo Strehl

Maffei, Helga Verena Leoni

Malafaia, Osvaldo

Maluf Filho, Fauze

Martino, Rodrigo Bronze de

Matheus, André Siqueira

Mattar, Rejane

Mattos, Angelo Alves de

Mattos, Angelo Zambam de

Mello, Catia Rejania de

Miszputen, Sender Jankiel

Montero, Edna Frasson de Souza

Moraes Filho, Joaquim Prado P de

Morais, Mauro Batista de

Moreira Jr, Hélio

Mota, Carolina Frade M. G. Pimentel

Moura, Eduardo Guimarães Hourneaux de

Nahas, Sergio Carlos

Nasi, Ary

Netinho, João Gomes

Nita, Marcelo Eidi

Ogata, Silvio Kazuo

Oksman, Alexandre
Oliveira e Silva, Adávio de

Oliveira, Claudia Pinto Marques de

Oliveira, Ricardo Brandt de

Ono, Suzane Kioko

Pajecki, Denis

Paraná, Raymundo

Pardini, Fernando

Pareja, José Carlos

Parise, Edison Roberto

Paschôa, Adilson Ferraz

Passos, Maria do Carmo Friche

Penteado, Sonia

Perez, Rodrigo Oliva

Pessoa, Mário Guimarães

Pizão, Paulo Eduardo

Poletti, Paula Bechara

Porta, Gilda

Pugliese, Renata

Quilici, Flávio Antonio

Quireze Jr, Claudemiro

Ramos Jr, Odery

Regadas, Sthela Maria Murad

Rezende Filho, Joffre

Ribeiro Jr, Ulysses

Rodrigues, Marcelo Bordalo

Rodrigues, Tomás Navarro

Rosa, Heitor

Rossini, Lucio Giovanni Battista

Sacco, Andrea Baldi de Freitas

Sakai, Paulo

Sallum, Rubens Antônio Aissar

Sampaio, Luiz Carlos Souza

Santo, Marco Aurelio

Santos, Vinicius

Sdepanian, Vera Lucia

Silva, Aloísio Souza Felipe

Silva, Roberta Gonçalves da

Silveira, Themis Reverbel da

Soares, Rosa Leonôra Salerno

Sobrado, Carlos Walter

Soifer, Luis

Speranzini, Manlio Basilio

Souza, Evandro de Oliveira

Strauss, Edna

Tacla, Mounib

Tahan, Soraia

Tovo, Cristiane Valle

Troncon, Luiz Ernesto de Almeida

Vinagre, Ruth Maria Dias Ferreira

Utiyama, Shirley Ramos da Rosa

Waisberg, Jaques

Waitzberg, Dan

Zaltman, Cyrla

Zantut, Luiz Fernando Corrêa

Zeitune, José Murilo Robilotta

Zilberstein, Bruno 